\title{
Reconfigurable nonreciprocity with a nonlinear Fano diode
}

\author{
$\mathrm{Yi} \mathrm{Xu}^{1,2}$ and Andrey E. Miroshnichenko, ${ }^{3, *}$ \\ ${ }^{1}$ School of Physics and Electronic Engineering, Guangzhou University, Guangzhou 510006, People's Republic of China \\ ${ }^{2}$ Department of Electronics, College of Information Science and Technology, Jinan University, Guangzhou 510632, People's Republic of China \\ ${ }^{3}$ Nonlinear Physics Centre, Research School of Science and Engineering, Australian National University, Canberra ACT 0200, Australia
}

(Received 11 November 2013; revised manuscript received 21 March 2014; published 21 April 2014)

\begin{abstract}
We propose a dynamically tunable nonreciprocal response for wave propagations by employing nonlinear Fano resonances. We demonstrate that the transmission contrast between waves propagating in opposite directions can be controlled by an excitation signal. In particular, the unidirectional transmission can be flipped at different time of a pulse, resembling a Fano diode with dynamical reconfigurable nonreciprocity. The key mechanism is the interaction between a linear and a nonlinear Fano resonance that allows for the tunable unidirectional wave propagation and ultrahigh transmission contrast ratio. We further present a realistic photonic example which demonstrates the properties of nonreciprocity can be dynamically manipulated using a pump pulse, based on the general theoretical model.
\end{abstract}

DOI: 10.1103/PhysRevB.89.134306

PACS number(s): 05.45.-a, 42.65.Pc

\section{INTRODUCTION}

Electronic diodes are one of the key elements in modern electronic devices, and even play an important role in our daily lives. The most common function of a diode is to allow an electric current to pass in one direction, while blocking current in the opposite direction, thus enabling the unidirectional current flux and the rectification of an electrical signal. To date, many contributions have been made for the rectification of different types of energy flows. For example, thermal diode for thermal flow (see Ref. [1], and references therein), acoustic diode for sonic wave [2,3], and electromagnetic diode for electromagnetic wave [4-12] have been demonstrated both theoretically and experimentally. As a particular example, violation of the Lorentz reciprocity in optical system prevents the light from retracing its directional transmission. It would facilitate certain potential applications, such as optical diodes and isolators [13]. There are several mechanisms which would lead to the breaking of reciprocity, such as the magneto-optical effect [12,14-16], nonlinear effect [4-10,17], and structure with time-dependent refractive index $[18,19]$. High-speed and on-chip optical circuits would require all-optical modulation, in which using nonlinear optical effects of the system could be one of the solutions [10]. More precisely, a low-power and high-contrast all-optical diode based on nonlinear photonic crystal is experimentally demonstrated recently [9].

A reconfigurable light-driven isolator attracts much attention due to its capacity of switching on/off the interband transition, which manifests itself as the transition from the nonreciprocal to reciprocal response, by using a control light beam [20]. Recently, an interesting phenomenon named reversible optical nonreciprocity was proposed where a nonreciprocal response can be flipped from transmitting a signal predominantly in one direction to the opposite one [21]. However, the contrast of such a system is low due to the limited scattering channel, and the possibility of dynamical manipulation has not been addressed yet. By offering the possibility for dynamically tuning of the nonreciprocity, one can direct the energy flow in real time and thus realize

*andrey.miroshnichenko@anu.edu.au more advanced control of the wave propagating systems. For example, diodes with tunable forward and backward transmission rates were proven to be significant in manipulating the properties of the wave rectification [11]. Furthermore, if the properties of nonreciprocity can be controlled instantaneously, a lot of basic devices, such as switch, router, and rectifier, etc., can be realized. Therefore, realizing the reconfigurable nonreciprocity is very crucial for precise wave manipulation, as is demonstrated in reconfigurable photonic crystal $(\mathrm{PhC})$ circuits [22].

One of the best ways for achieving high-transmission contrast can rely on Fano resonances, where the transmission can completely vanish due to the destructive interference [23-26]. Diodes based on Fano resonances may benefit from their typical resonant reflection. Therefore, rectification with ultrahigh-contrast ratio can be realized [27]. It was shown that coupling two nonlinear Fano defects can induce the symmetry breaking of the system [28]. Such a system reveals rich nonlinear dynamics and thus facilitates one's manipulation of the optical bistable scattering [29,30]. In this paper, we suggest that an even simpler system, consisting of a waveguide with two side-coupled Fano defects, one is with cubic nonlinearity while the other one is linear, exhibits quite interesting physical property, namely, dynamical reconfigurable nonreciprocity (DRN). It means that by modulating the input signal or adding a pump pulse, we can dynamically change the predominant transmission direction and transmission contrast between reversal incident signal. We demonstrate that the interaction between a linear and a nonlinear Fano resonance has a pronounced DRN. It is shown that the unidirectional transmission of nonreciprocity not only can be manipulated by choosing the operating frequency and geometry parameters, but also can be dynamically controlled by modulating the input signal. These properties are different from obtaining an asymmetry nonlinear response in a structure with asymmetric nonlinear elements.

\section{MODEL}

The transmission properties of the proposed nonreciprocal system shown in Fig. 1 can be studied by using the modified 


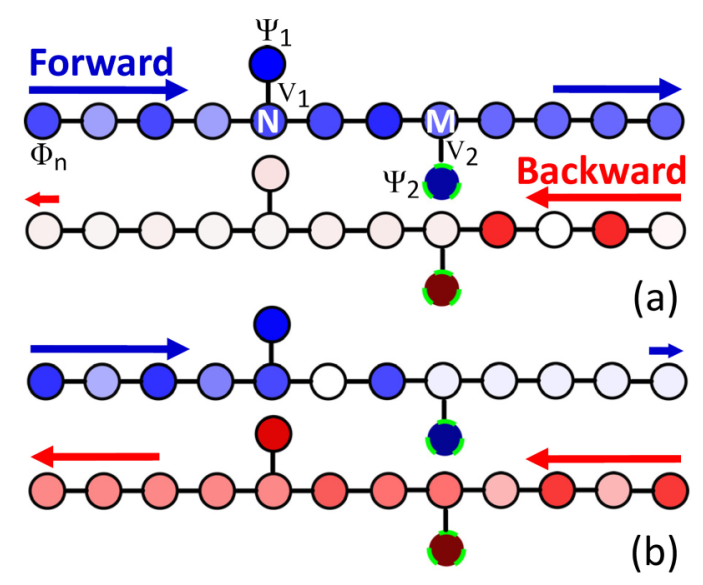

FIG. 1. (Color online) Schematic view of the reversible nonreciprocal response based on the nonlinear Fano resonance. The arrows indicate the direction of the forward and backward transmission. The same system can support waves propagation predominantly either in one (a) or opposite (b) direction for different excitation signals. The color scale stands for the intensity of the field in each cite which are two particular solutions of Eq. (1). Nonlinear Fano defect is shown by a dashed ring while others are linear. Forward transmission means that the injection is from the left side, while backward transmission is corresponding to the excitation from the right side, as are indicated by the big arrows.

Fano-Anderson model [31]. It allows us to derive analytical solutions of the nonlinear transmission at reversal incident directions. The equations describing the nonlinear dynamics of the scattering are

$$
\begin{aligned}
& i \dot{\phi}_{n}=C\left(\phi_{n-1}+\phi_{n+1}\right)+\delta_{n, N} V_{1} \psi_{1}+\delta_{n, M} V_{2} \psi_{2}, \\
& i \dot{\psi}_{1}=V_{1} \phi_{N}+E_{d 1} \psi_{1}, \\
& i \dot{\psi}_{2}=V_{2} \phi_{M}+E_{d 2} \psi_{2}+\lambda\left|\psi_{2}\right|^{2},
\end{aligned}
$$

where the overdot stands for the derivative in time, $\phi_{n}$ and $\psi_{1,2}$ represent the complex fields of the sites in the chain and sidecoupled defects, respectively. $E_{d 1, d 2}$ are the defect energy, $\lambda$ is the nonlinearity parameter, $C$ is the nearest-neighbor coupling constant, $\delta_{n m}$ is the Kronecker delta symbol, and $V_{1,2}$ are the side-coupled strength between defects and the chain. $N, M$ are used to specify the distance between two side-coupled defects, as is indicated in the upper panel of Fig. 1(a). The transmission matrix for the system, where $\varepsilon_{1,2}=V_{1,2}^{2} /(\omega-$ $\left.E_{d 1, d 2}\right), \omega=2 C \cos q$, and $C_{q}=2 C \sin q$, is shown in Eq. (2) with $\phi_{n}=A_{n} e^{-i \omega t}$ and $\psi_{1,2}=B_{1,2} e^{-i \omega t}, A_{n}$ and $B_{1,2}$ are complex numbers [31]. The input signal is fed either from the right side of the chain or the left side of the chain. For the excitation from the left side, $A_{n}=I e^{-i q n}+r e^{i q n}$

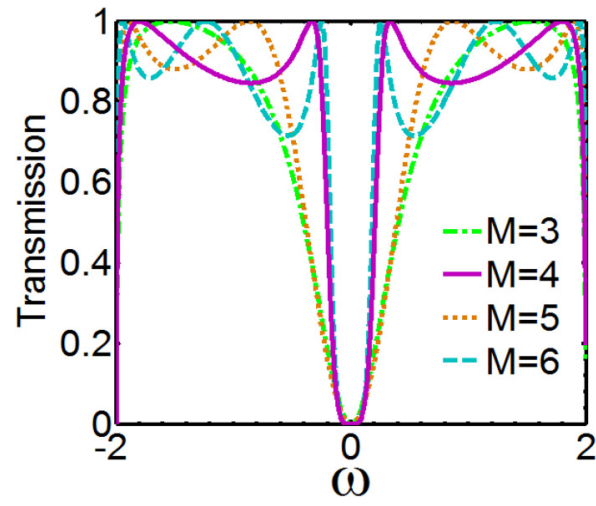

FIG. 2. (Color online) Linear transmission of the system presented in Fig. 1. Without loss of generality, we put here $N=1$, $E_{d 1, d 2}=0$, and $V_{1,2}=0.6$.

with $n<N$, where $I$ and $r$ represent the incoming and reflected wave amplitudes far from the side-coupled defect sites. Linear transmission of the system can be obtained by $T=|1 / K(2,2)|^{2}$.

$$
\begin{aligned}
K= & \frac{-1}{C_{q}^{2}}\left[\begin{array}{cc}
\varepsilon_{1}+i C_{q} & \varepsilon_{1} e^{-i 2 N q} \\
-\varepsilon_{1} e^{i 2 N q} & -\varepsilon_{1}+i C_{q}
\end{array}\right] \\
& \times\left[\begin{array}{cc}
\varepsilon_{2}+i C_{q} & \varepsilon_{2} e^{-i 2 M q} \\
-\varepsilon_{2} e^{i 2 M q} & -\varepsilon_{2}+i C_{q}
\end{array}\right] .
\end{aligned}
$$

Four representative examples are plotted in Fig. 2. As can be seen from these results, when $M-N=2 n+1$ and $n=$ $1,2,3 \ldots$, the transmission forms a nearly flat bottom stop band with sharp edge near the Fano resonances of the sidecoupled defects.

When we add a cubic nonlinearity to one of the Fano defects, the reciprocity can be broken for the input power above certain threshold. Moreover, the indirect interaction between the linear and nonlinear Fano defects via the chain suggests the possibility of the reversible nonreciprocal effect [21]. In particular, the incident wave propagating in opposite directions would excite the nonlinear Fano defect with different rates, as is shown in Fig. 1. Because the nonlinear Fano resonance is input power dependent [31], different power injection would lead to a distinct shift of the nonlinear Fano resonance, which, in turn, would interact differently with the linear Fano resonance. Furthermore, the bistability of the nonlinear Fano resonance offers another opportunity to control the interaction by dynamically choosing different branches in the hysteresis loop of the bistable state [31]. Such a tunable interaction gives rise to the possibility for dynamically manipulating the physical properties of wave scattering. The nonlinear transmission of the system can be written as follows:

$$
T_{L R / R L}=\frac{\alpha_{q 1}^{2} x^{2}}{2(1-\cos 2 q l)\left(1-\alpha_{q 1} x\right)+\alpha_{q 1}^{2} x^{2}-2 \sin (2 q l)\left(\alpha_{q 1}+x\right)+\left(\alpha_{q 1}+x\right)^{2}},
$$

where $\alpha_{q 1, q 2}=C_{q}\left(\omega-E_{d 1, d 2}\right) / V_{1,2}^{2}, l=M-N$, and $x=$ $-\cot \theta, \theta$ is the scattering phase of the nonlinear Fano defect, while $x$ is a real solution of the following cubic equation for the elastic scattering problem [32]:

$$
a x^{3}+b x^{2}+c x+d_{L R / R L}=0
$$




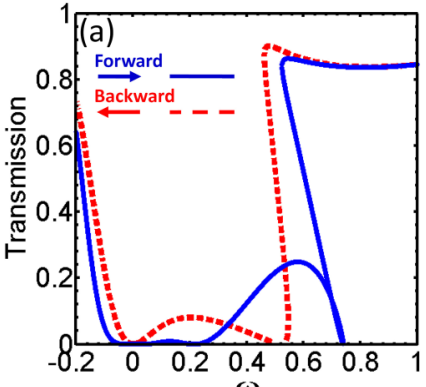

(i)

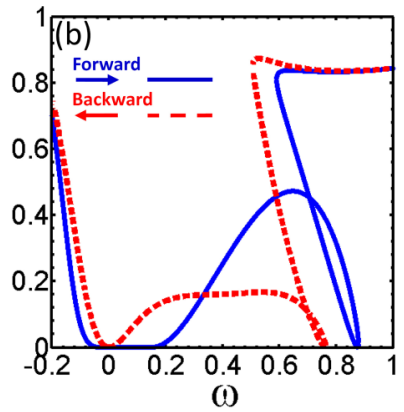

FIG. 3. (Color online) (a) Nonlinear transmission of opposite incident directions. Here $N=1, M=4, E_{d 1, d 2}=0, V_{1,2}=0.6$, $\lambda=1$, and incident power $|I|^{2}=0.05$. (b) The same plot as (a) except for $|I|^{2}=0.08$. Solid blue/dashed red color indicates the forward/backward incident direction throughout this paper, as is indicated in Figs. 1 and 3.

with $\quad a=\alpha_{q 1}^{2}+1, \quad b=(2 \cos 2 q l+1) \alpha_{q 1}-2 \sin 2 q l-$ $\alpha_{q 2}\left(\alpha_{q 1}^{2}+1\right), \quad c=2-2 \cos 2 q l-2 \alpha_{q 1} \sin 2 q l+\alpha_{q 1}^{2}-$ $2 \alpha_{q 2}\left(\alpha_{q 1} \cos 2 q l-\sin 2 q l\right), \quad d_{L R}=-\alpha_{q 2} \gamma+\lambda C_{q}^{3} \alpha_{q 1}^{2} I^{2} / V_{2}^{4}$ and $d_{R L}=-\alpha_{q 2} \gamma+\lambda C_{q}^{3} \alpha_{q 1}^{2} I^{2} \eta / V_{2}^{4}, \quad \gamma=2-2 \cos 2 q l-$ $2 \alpha_{q 1} \sin 2 q l+\alpha_{q 1}^{2}$, and $\eta=\left|e^{3 i q}-\omega V_{1}^{2}+V_{1}^{2} / \omega\right|^{2}$ for the cases $l=3$ and $C=1$. Here, $L R$ means the forward transmission (blue color) and $R L$ denotes the backward case (red color) as are indicated in Fig. 1(a).

As can be seen from the Eq. (4), the asymmetry scattering of the system arises when $d_{L R} \neq d_{R L}$ and thus different roots of the cubic equation. Intuitively, such asymmetry $(\eta \neq 1)$ can be fulfilled by choosing suitable frequency $\omega$ and coupling strength $V_{1}$. Figures 3(a) and 3(b) are two examples with different input powers $|I|^{2}=0.05$ and 0.08 , respectively. Other parameters can be found in the caption. It can be seen from these figures that the system exhibits asymmetric response for opposite incident directions. Strict one-way transmission with high contrast only occurs at the nonlinear
Fano resonances, which is similar to the case presented in Ref. [27]. Furthermore, given certain $\omega$ and $V_{1}$ preserving $\eta \neq 1$, the input power $I^{2}$ dependence of both $d_{L R}$ and $d_{R L}$ offers us another degree of freedom to realize advanced manipulation of the nonreciprocity. When $d_{L R} \neq d_{R L}$, the nonlinear Fano resonances of the system occur at different input power for reversal incident directions. Therefore, ultrahighcontrast-ratio Fano diodes with reversible nonreciprocity are presented at these two corresponding transmission dips, as shown in Figs. 4(a)-4(d). Figure 4(a) presents the dependence of the forward and backward transmissions on the input power when $\omega=0.28$. There are distinct transmissions for reversal incident waves. Figure 4(b) shows the corresponding $T_{L R} / T_{R L}$ in Fig. 4(a). These results show that we can flip the unidirectional transmission by simply controlling the input power. Figures 4(c) and 4(d) show the case when we use a suitable frequency detuning $\omega=0.45$, i.e., different $\eta$, to trigger bistability. Changing the input power is corresponding to tune the interaction between the linear and nonlinear Fano resonances into a distinct bistable state. Thus, the rectification capacity of the system can be manipulated. It is demonstrated that the input power dependence of $T_{L R} / T_{R L}$ can be engineered from tunable single value to tunable bistability by choosing different $\eta$, which would facilitate the advanced control of the rectification, as are shown in Figs. 4(b) and 4(d).

It is demonstrated that the nonlinear Fano defect suffers from modulational instability near the resonance under a continuous-wave excitation [33], which resembles the scenario of wave scattering by a nonlinear center first addressed in Ref. [34]. The stability analysis of steady-state solutions shows that the intermediate state between two bistable states are unstable, as is shown by the dynamical results in Fig. 5(b). The modulation instability of our system is similar to the previous reported one [33]. So, there is still a way to access the bistable hysteresis loop by a Gaussian pulse with a suitable duration. The Crank-Nicolson method [35] with absorption

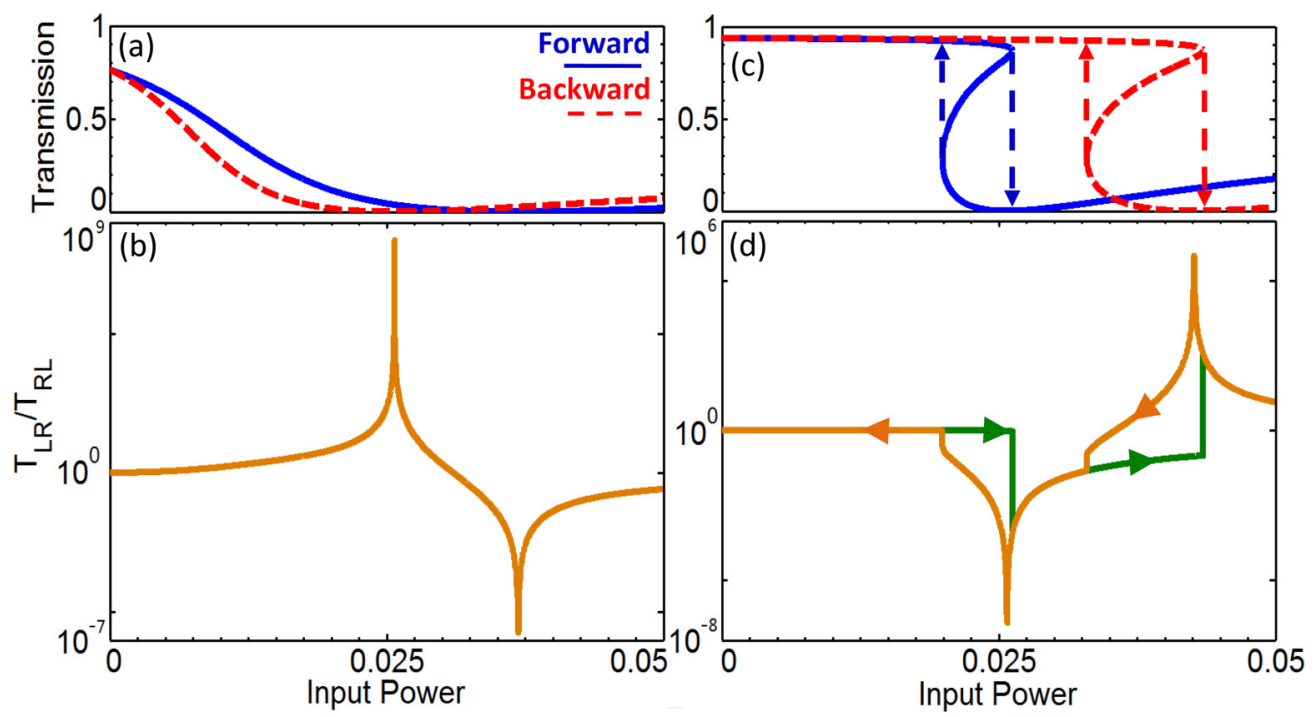

FIG. 4. (Color online) (a) The dependence of nonlinear transmission on the input power of the system for opposite incident directions (frequency $\omega=0.28$ ). Here, $N=1, M=4, E_{d 1, d 2}=0, \lambda=1$, and $V_{1,2}=0.6$. Solid lines represent the forward excitation, while dashed lines indicate the backward one. (b) The corresponding $T_{L R} / T_{R L}$ contrast ratio versus input power in (a). (c), (d) The same plots as (a) and (b) except for $\omega=0.45$. (a)-(d) share a similar $x$ label. The arrows indicate the transition directions between two stable states. 

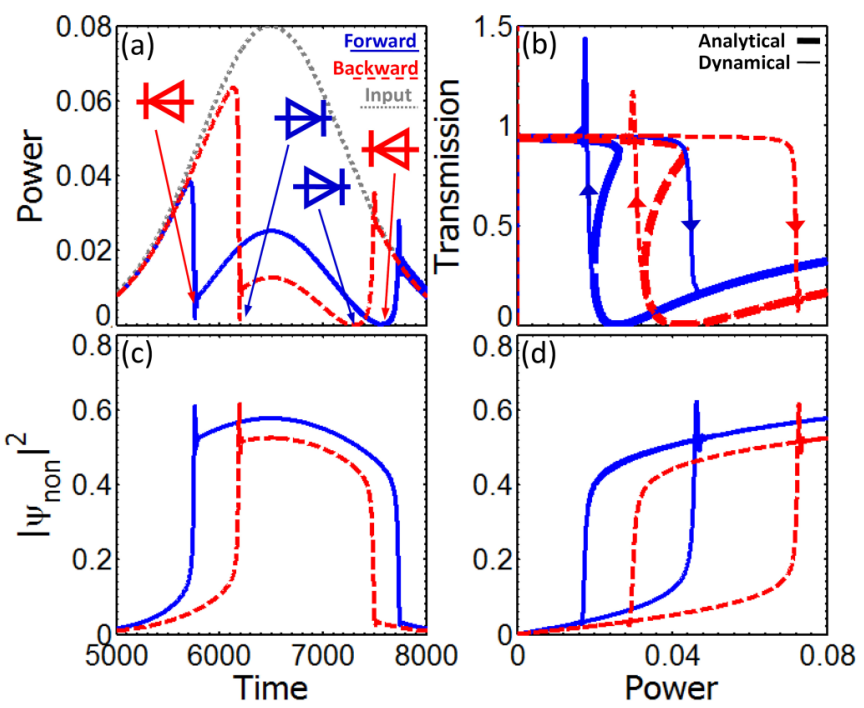

FIG. 5. (Color online) (a) Dynamics of the system at reversal incident direction with a Gaussian pulse with $I=I_{0} \exp [-(t-$ $\left.\left.t_{0}\right)^{2} / W^{2}\right] \sin (\omega t)$, where $W=1400$ and $\omega=0.14$. The geometry parameters are similar with those in Fig. 4(c). The forward directions of the diode at specified pulse times are indicated by the insets. (b) Transmissions (thin lines) derived from (a) and the corresponding analytical results (thick lines). (c) Time evolutions of the nonlinear cavity excitations. (d) The effective pumping rates of the cavity. Solid lines stand for the forward excitation, while dashed lines represent the backward case.

boundary condition [36] is used to solve Eq. (1). Figures 5(a) and $5(\mathrm{c})$ present the dynamics of the system under the excitation of a pulse where we only show part of pulse for better visualization. As can be seen from the marked forward direction of the diode in Fig. 5(a), the unidirectional transmission of the nonreciprocity is dynamically reconfigured at a different time of the pulse's rising and falling edges, respectively. It is because the threshold powers, which trigger the transition between two stable states in the cases of the increasing and decreasing input power, are different referring to reversal incident waves, as is shown in Fig. 5(b). They validate that the DRN could be realized by shaping the excitation condition to switch between the high-transmission states and the nonlinear Fano resonant low-transmission state. Figure 5(b) also provides the analytical results (thick curves) which agree with the dynamical simulations (thin curves). The oscillation between two bistable states indicates the transition between them and it is the property of the dynamical bistability [33]. Figures 5(c) and 5(d) present the dynamics of the power in the nonlinear Fano defect and the corresponding tunable power in the nonlinear cavity, which demonstrates the incident direction dependence of the pumping rate in the nonlinear Fano defect. It is consistent with the idea of which we use directional-dependent nonlinear Fano resonance to manipulate the wave transmission.

\section{PHOTONIC CRYSTAL REALIZATION}

Now, we provide a realistic example of the dynamical reconfigurable nonreciprocity which is consisted of a linear photonic crystal $(\mathrm{PhC})$ waveguide, with a side-coupled linear and nonlinear cavities pair. The $\mathrm{PhC}$ is formed by dielectric pillar rods arranged in a square lattice. The radius of the rods are $r=0.18 a$, where $a$ is the lattice constant and the refractive index $n=3.4$. Air is the background medium. The waveguide is created by removing one row of rods. The nonlinear defect on the right is made by a polymer rod with $r_{d}=0.1 a, n_{l}=$ $1.6, n_{2}=1.14 \times 10^{-12} \mathrm{~cm}^{2} / \mathrm{W}$, and $n=n_{l}+n_{2} I$, while the linear defect is introduced by removing one dielectric rod. The nearest rods of the linear defect are shifted $0.028 a$ away from the center to keep the eigenfrequencies of two defects the same and thus obtain flat stop band with sharp
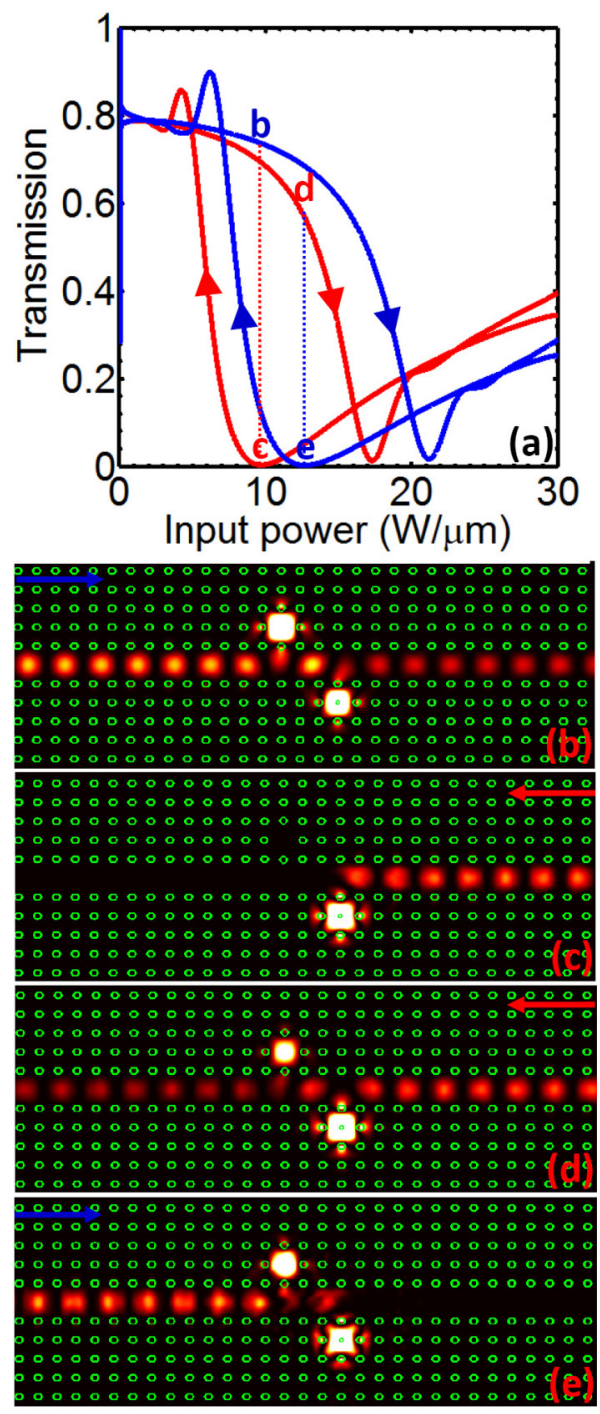

FIG. 6. (Color online) (a) The FDTD results of the nonlinear transmission at reversal incident directions. A Gaussian pulse with a carrier frequency $f=0.3782 \pi c / a$ and 20-ps duration is used as an input probe signal. (b)-(e) are the corresponding instantaneous electric field $|E|^{2}$ distribution marked b-e in (a), respectively. The structure details of the PhCs are outlined by the green lines. The side-coupled cavity on the right is with Kerr nonlinearity. (b)-(e) are normalized with each maximum and are saturated for better visualization. The FDTD grid is nonuniform. The grid size near the defect is $a / 100$ to tell the small feature, while the others are $a / 50$. The computation domain is surrounded by perfect matching layers to absorb outgoing wave. 


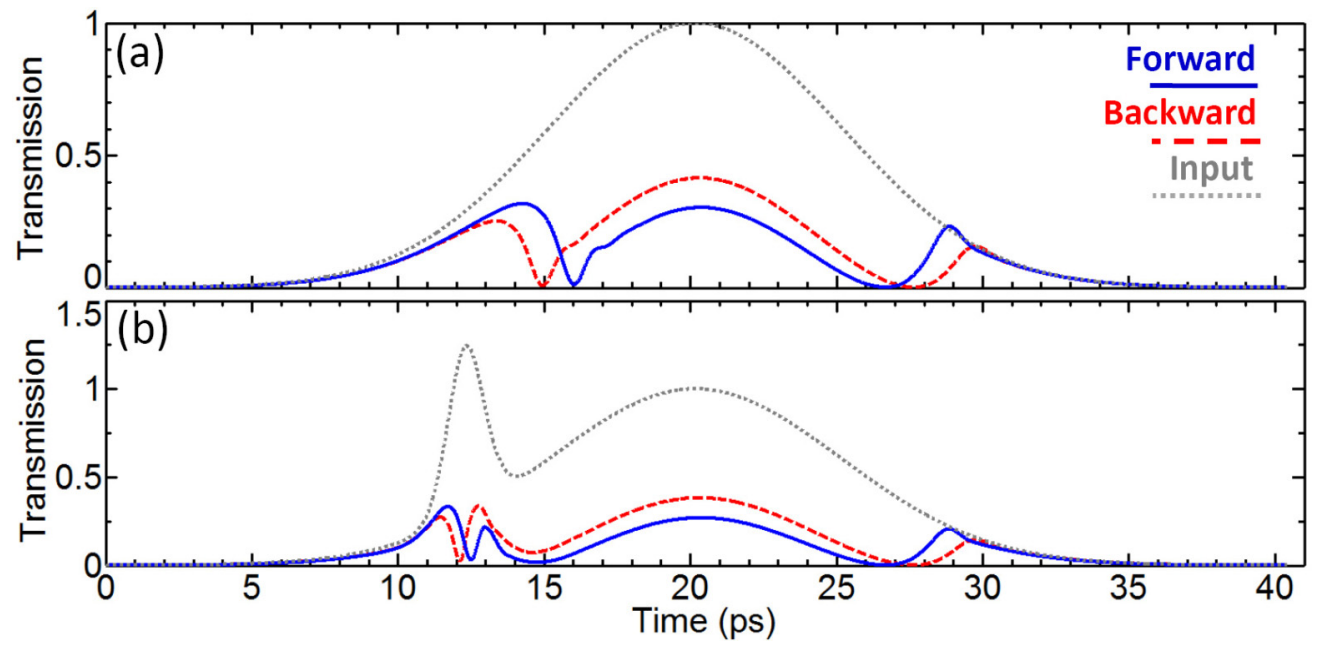

FIG. 7. (Color online) (a) The FDTD results of the pulse dynamics at reversal incident directions $(\omega=0.3782 \pi c / a)$. The input signal, which is used as a probe pulse to access the bistability, is shown by the dotted line. (b) Reconfigurable nonreciprocity driven by a pump pulse whose duration is comparable to the nonlinear cavity's lifetime. The maximum amplitude of the pump pulse is one third of the probe one. The pump pulse is superimposed upon the input Gaussian pulse, as is shown by the dotted line in (b). The carrier frequency of the pump pulse is the same as the probe pulse [23].

edge. The structure details are outlined by the green lines in Figs. 6(b)-6(e). It was demonstrated that the nonlinear dynamics of this photonic structure can be described by a discrete model similar to the one presented in Sec. II [37]. At the same time, it should be mentioned that the Fano-Anderson model can be used to exactly model the optical response of the $\mathrm{PhC}$ waveguide and cavity system shown in Figs. 6(b)-6(e), providing that the Green's function of the two-dimensional periodic structure without defects is obtained numerically together with the nonlocal effect and long-range interaction are taken in to account. In this paper, we use the simplified Fano-Anderson model, which is proved to be capable of catching the essential physics of nonlinear dynamics in $\mathrm{PhC}$ waveguide and cavity-coupling system [33], to analytically study the reconfigurable nonlinear scattering. It could provide a clear physical picture for the reconfigurable nonreciprocity, which is similar as the one used in Ref. [17].

The finite difference time domain (FDTD) simulation results [see Figs. 6(a) and 7(a)] obtained by a Gaussian pulse show good agreement with the theoretical model. They prove that we can dynamically manipulate the nonreciprocity of the system by using the rising and falling edges of a pulse with suitable duration. The flipping of unidirectional transmission is indicated by b,c and d,e in Fig. 6(a), in which the forward direction of the Fano diode is reversed. We can use the pump-assisting method to access the low-transmission cases $\mathrm{c}$ and e marked in Fig. 6(a) $[7,23]$. The corresponding instantaneous electric field distributions $|E|^{2}$ in the dynamic manipulation of the DRN are shown in Figs. 6(b)-6(e). They manifest themselves as distinct interfering effects because different modes of the system are excited. For the Fano diode blocking the forward propagating wave, the interaction between two Fano defects is crucial [see Fig. 6(e)], while the nonlinear Fano defect dominates the resonant reflection when the forward direction is flipped [see Fig. 6(c)]. The input power needed to flip the forward direction of the diode is shown by the horizontal label in Fig. 6(a). FDTD modeling detail can be found in the caption of Fig. 6. Furthermore, launching a suitable pump pulse (duration is comparable to the nonlinear cavity's lifetime) together with the probe one, it is possible to change the properties of the nonreciprocity. Because of distinct nonlinear feedbacks obtained at reversal excitations, their corresponding transmissions drop at different time referring to the same pump pulse, as is shown in Fig. 7(b). We can conclude from Figs. 6 and 7 that the nonreciprocity of the nonlinear Fano resonance system has distinct scenarios with respect to the input signal for reversal incident waves, which provides the possibility of dynamical wave manipulation.

It should be pointed out that the proposed Fano diode can be realized in membrane-type $\mathrm{PhC}$ too. The linear $\mathrm{PhC}$ cavity can be mimicked by the air-slot mode-gap confined $\mathrm{PhC}$ nanocavity [38]. The loss effect including the scattering loss and the absorption loss can be modelled by replacing $\omega \rightarrow \omega+\alpha_{\text {loss }} i$ [39]. These kinds of loss would broaden the linewidth of the Fano resonance and thus increase the power for switching. Using state-of-the-art nanotechnology to release these optical losses, the physical concept of dynamical reconfigurable nonreciprocity can be preserved.

\section{CONCLUSIONS}

We demonstrate the dynamical reconfigurable nonreciprocity based on a nonlinear Fano resonance system. Tunable rectification, which manifests as the tunable bistability and the dynamical reconfiguration of diodes forward direction, is theoretically investigated. The interaction between the linear and nonlinear Fano resonances plays an important role in manipulating the nonreciprocity. Numerical experiments confirm our motivation via a realistic photonic example. Our results could pave the way for the advanced manipulation of wave rectification. Because of the similarity between the modified Fano-Anderson model and the discrete nonlinear Schrödinger equation which is shown to be relevant in many other physical contexts, we believe our results can be generalized to similar physical systems such as the coupled 
ring resonators system [17], the coupled optical waveguide arrays system [40], and many others.

\section{ACKNOWLEDGMENTS}

The work of A. E. Miroshnichenko was supported by the Australian Research Council through Future Fellowship program (No. FT110100037). Y. Xu acknowledges the support from National Natural Science Foundation (Grant No. 11304047), Foundation for Distinguished Young Talents in Higher Education of Guangdong Province (Grant No. 2013LYM0067), Natural Science Foundation of Guangdong Province (Grant No. S201310014807), and the Science Foundation of Guangzhou University (Grant No. XY051001/2001).
[1] N. Li, J. Ren, L. Wang, G. Zhang, P. Hänggi, and B. Li, Rev. Mod. Phys. 84, 1045 (2012).

[2] V. F. Nesterenko, C. Daraio, E. B. Herbold, and S. Jin, Phys. Rev. Lett. 95, 158702 (2005).

[3] S. Lepri and G. Casati, Phys. Rev. Lett. 106, 164101 (2011).

[4] M. Scalora, J. P. Dowling, C. M. Bowden, and M. J. Bloemer, J. Appl. Phys. 76, 2023 (1994).

[5] S. F. Mingaleev and Y. S. Kivshar, J. Opt. Soc. Am. B 19, 2241 (2002).

[6] M. W. Feise, I. V. Shadrivov, and Y. S. Kivshar, Phys. Rev. E 71, 037602 (2005).

[7] X. S. Lin, J. H. Yan, L. J. Wu, and S. Lan, Opt. Express 16, 20949 (2008).

[8] I. V. Shadrivov, V. A. Fedotov, D. A. Powell, Y. S. Kivshar, and N. I. Zheludev, New J. Phys. 13, 033025 (2011).

[9] X. Y. Hu, Z. Q. Li, J. X. Zhang, H. Yang, Q. H. Gong, and X. P. Zhang, Adv. Funct. Mater. 21, 1803 (2011).

[10] L. Fan, J. Wang, L. T. Varghese, H. Shen, B. Niu, Y. Xuan, A. M. Weiner, and M. H. Qi, Science 335, 447 (2012).

[11] J. Hwang, M. H. Song, B. Park, S. Nishimura, T. Toyooka, J. W. Wu, Y. Takanishi, K. Ishikawa, and H. Takezoe, Nat. Mater. 4, 383 (2005).

[12] L. Bi, J. J. Hu, P. Jiang, D. H. Kim, G. F. Dionne, L. C. Kimerling, and C. A. Ross, Nat. Photonics 5, 758 (2011).

[13] S. Fan, R. Baets, A. Petrov, Z. Yu, J. D. Joannopuolos, W. Freude, A. Melloni, M. Popovic, M. Vanwolleghem, D. Jalas, M. Eich, M. Krausse, H. Renner, E. Brinkmeyer, and C. R. Doerr, Science 335, 38 (2012).

[14] A. Davoyan, A. Mahmoud, and N. Engheta, Opt. Express 21, 3279 (2013).

[15] R. J. Potton, Rep. Prog. Phys. 67, 717 (2004).

[16] Z. Wang, Y. Chong, J. D. Joannopoulos, and M. Soljačić, Nature (London) 461, 772 (2009).

[17] F. Nazari, N. Bender, H. Ramezani, M. K. Moravvej-Farshi, D. N. Christodoulides, and T. Kottos, Opt. Express 22, 9574 (2014).

[18] Z. Yu and S. Fan, Nat. Photonics 3, 91 (2009).

[19] H. Lira, Z. Yu, S. Fan, and M. Lipson, Phys. Rev. lett. 109, 033901 (2012).

[20] M. S. Kang, A. Butsch, and P. St. J. Russell, Nat. Photonics 5, 549 (2011).

[21] A. E. Miroshnichenko, E. Brasselet, and Y. S. Kivshar, Appl. Phys. Lett. 96, 063302 (2010).
[22] C. Grillet, C. Monat, C. L. Smith, M. W. Lee, S. TomljenovicHanic, C. Karnutsch, and B. J. Eggleton, Laser Photon. Rev. 4, 192 (2010).

[23] M. F. Yanik, S. Fan, and M. Soljačić, Appl. Phys. Lett. 83, 2739 (2003).

[24] A. E. Miroshnichenko, S. Flach, and Yu. S. Kivshar, Rev. Mod. Phys. 82, 2257 (2010).

[25] B. Luk'yanchuk, N. I. Zheludev, S. A. Maier, N. J. Halas, P. Nordlander, H. Giessen, and C. T. Chong, Nat. Mater. 9, 707 (2010).

[26] M. Rahmani, B. Luk'yanchuk, and M. Hong, Laser Photon. Rev. 9, 707 (2012).

[27] W. Ding, B. Luk'yanchuk, and C. W. Qiu, Phys. Rev. A 85, 025806 (2012).

[28] B. Maes, P. Bienstman, and R. Baets, Opt. Express 16, 3069 (2007).

[29] E. N. Bulgakov and A. F. Sadreev, Phys. Rev. B 81, 115128 (2010).

[30] E. N. Bulgakov, K. Pichugin, and A. Sadreev, Phys. Rev. B 83, 045109 (2011).

[31] A. E. Miroshnichenko, S. F. Mingaleev, S. Flach, and Yu. S. Kivshar, Phys. Rev. E 71, 036626 (2005).

[32] H. J. Lipkin, Quantum Mechanics: New Approaches to Selected Topics (North-Holland, Amsterdam, 1973).

[33] A. E. Miroshnichenko, Yu. Kivshar, C. Etrich, T. Pertsch, R. Iliew, and F. Lederer, Phys. Rev. A 79, 013809 (2009).

[34] B. A. Malomed and M. Y. Azbel, Phys. Rev. B 47, 10402 (1993).

[35] J. Crank and P. Nicolson, Proc. Cambridge Philos. Soc. 43, 50 (1947).

[36] A. Arnold, M. Ehrhardt, and I. Sofronov, Commun. Math. Sci. 1, 501 (2003).

[37] S. F. Mingaleev and Y. S. Kivshar, Phys. Rev. Lett. 86, 5474 (2001).

[38] J. Gao, J. F. McMillan, M.-C. Wu, J. Zheng, S. Assefa, and C. W. Wong, Appl. Phys. Lett. 96, 051123 (2010).

[39] S. F. Mingaleev, A. E. Miroshnichenko, and Yuri S. Kivshar, Opt. Express 16, 11647 (2008).

[40] A. Szameit, I. L. Garanovich, M. Heinrich, A. A. Sukhorukov, F. Dreisow, T. Pertsch, S. Nolte, A. Tüennermann, and Yu. S. Kivshar, Nat. Phys. 5, 271 (2009). 